\title{
Resistant Maltodextrin Decreases Micellar Solubility of Lipids and Diffusion of Bile Salt Micelles and Suppresses Incorporation of Micellar Fatty Acids into Caco-2 Cells
}

\author{
Ikuo IKEDA ${ }^{1}$, Kyouhei TAMAKUni ${ }^{1}$, Tomomi SAKUMA ${ }^{1}$, \\ Ran OZAWA ${ }^{1}$, Nao INOUE $^{1}$ and Yuka Kishimoto ${ }^{2}$ \\ ${ }^{1}$ Laboratory of Food and Biomolecular Science, Graduate School of Agricultural Science, \\ Tohoku University, Sendai, Miyagi 981-8555, Japan \\ ${ }^{2}$ Matsutani Chemical Industry Co., Ltd., Itami, Hyogo 664-8508, Japan
}

(Received April 1, 2016)

\begin{abstract}
Summary Several studies have suggested that resistant maltodextrin (RMD) suppresses intestinal lipid absorption in experimental animals and humans. However, possible mechanisms underlying this effect are not known. In this study, effects of RMD on processes of the absorption of various lipids were investigated in vitro. RMD dose-dependently suppressed the solubility of various lipid components, including 1-mono-oleoylglycerol, oleic acid, and phosphatidylcholine in bile salt micelles in vitro. When the diffusion rate of bile salt micelles through a filter membrane was investigated in vitro, bile salt micelles containing RMD diffused more slowly than those without RMD. Incorporation of $\left[1-{ }^{14} \mathrm{C}\right]$ oleic acid into Caco-2 cells from the RMD-containing bile salt micelles was significantly smaller than that from the control micelles (without RMD). These results show that RMD suppresses intestinal absorption of lipids by decreasing their micellar solubility and the diffusion rate of bile salt micelles. Key Words resistant maltodextrin, lipid absorption, bile salt micelles, Caco-2 cells
\end{abstract}

Because postprandial hypertriacylglycerolemia is one of the risk factors of coronary heart disease (1), its prevention and suppression may be important for human health. Given that the postprandial phase is characterized by the presence of plasma triacylglycerol-rich lipoproteins of intestinal origin (1), attenuation of dietary fat absorption could be an effective way to prevent postprandial hypertriacylglycerolemia.

Resistant maltodextrin (RMD) is a low-viscosity viscous soluble dietary fiber and is produced by a combination of heat and enzymatic treatment of cornstarch (2). RMD is a mixture of short-chain polymers of glucose with $\alpha-1,2$ and $\alpha-1,3$ glucosidic bonds in addition to $\alpha-1,4$ and $\alpha-1,6$ bonds (Fig. 1). Research has shown that dietary RMD can decrease serum cholesterol (3) and triacylglycerol (TAG) concentrations (4). It has been suggested that these functions can be ascribed to its suppressive effects on dietary lipid absorption $(3,4)$. Recently, Kishimoto et al. (5) reported that postprandial blood TAG elevation is significantly suppressed in rats treated orally with a fat emulsion containing RMD and in humans given RMD after a meal. They also showed that dietary RMD increases fecal excretion of fat in rats and humans without inhibiting pancreatic lipase activity (6). The results suggest that RMD suppresses lipid absorption in rats and humans. This can be a strategy to suppress postprandial serum TAG concentration. However, the precise mechanisms of the suppressive effects of RMD on lipid absorption are not clear.

It is known that hydrolyzed products of lipids are incorporated into bile acid micelles in the small intes-

E-mail: iikeda@biochem.tohoku.ac.jp tine, and the micelles diffuse to the intestinal epithelial cell surface through the unstirred water layer (7). Thus, the solubilization of these components in bile salt micelles and the rate of diffusion of bile salt micelles are crucial factors for lipid absorption and digestion. We hypothesized that these factors can be involved in the suppressive effect of RMD on TAG absorption.

In the present study, we explored possible mechanisms underlying this effect by examining the effects of RMD on micellar solubility of lipids, on diffusion of bile salt micelles, and on fatty acid uptake in differentiated Caco- 2 cells.

\section{MATERIALS AND METHODS}

Chemicals. 1-Mono-oleoylglycerol (MO), oleic acid, trioleoylglycerol (TO), phosphatidylcholine (PC), cholesterol, pentadecanoic acid, egg lecithin, and $5 \alpha$-cholestane were purchased from Sigma-Aldrich (Tokyo, Japan). 23-Nor-5 $\beta$-cholanic acid was acquired from Steraloids (Newport, RI). Sodium taurocholate was purchased from Nacalai Tesque, Inc. (Kyoto, Japan). Glycerin was purchased from Wako Pure Chemical Industries, Ltd. (Tokyo, Japan). $\left[1-{ }^{14} \mathrm{C}\right]$ Oleic acid was purchased from Amersham Pharmacia Biotech (Tokyo, Japan). High oleic safflower oil is a product of Nisshin Oillio (Tokyo, Japan). RMD (Fibersol-2, average molecular weight $=1,800$ ) (6) and maltodextrin (average molecular weight $=1,800)$ are products of Matsutani Chemical Industry (Hyogo, Japan). The dietary fiber content of RMD and maltodextrin used in these experiments was $91.9 \%$ and $0 \%$, respectively.

Preparation of the lipid emulsion and isolation of bile salt micelles in vitro. The solubility of lipids in bile salt 


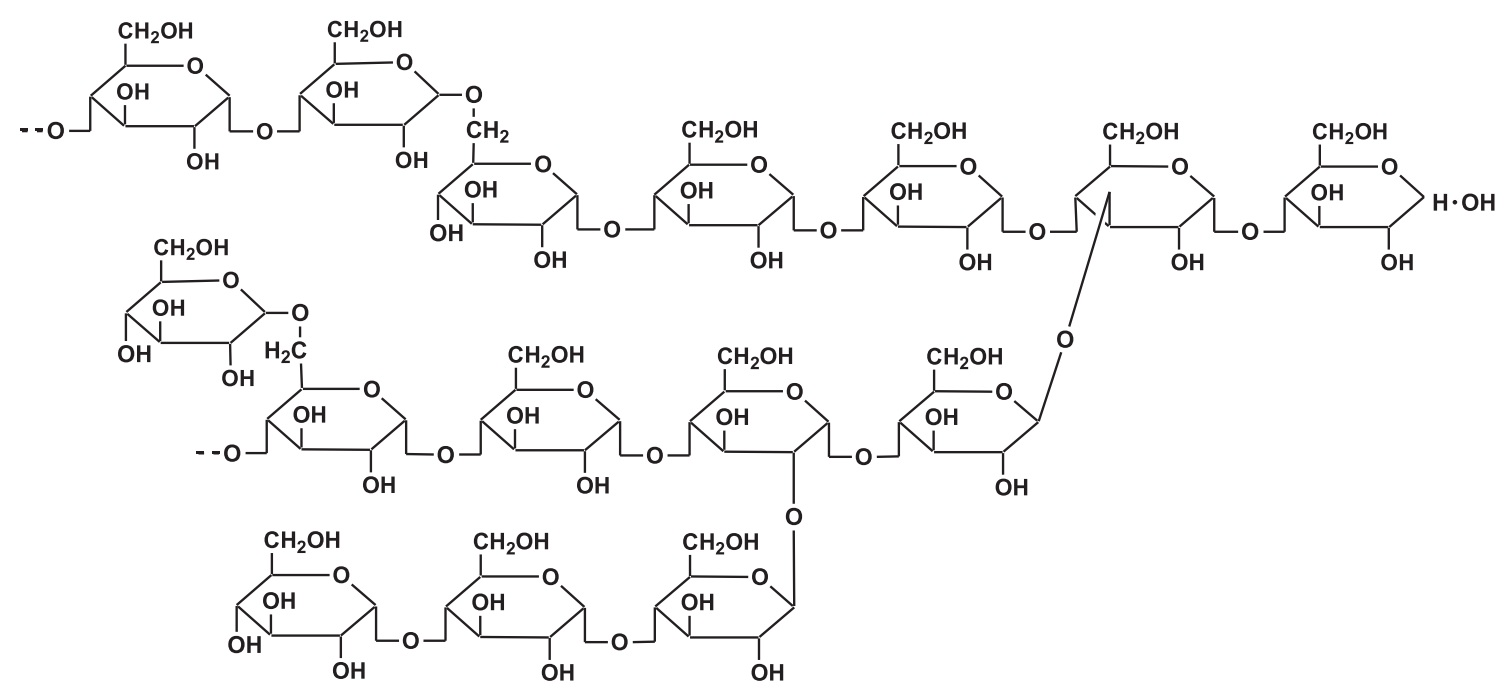

Fig. 1. Chemical structure of resistant maltodextrin.

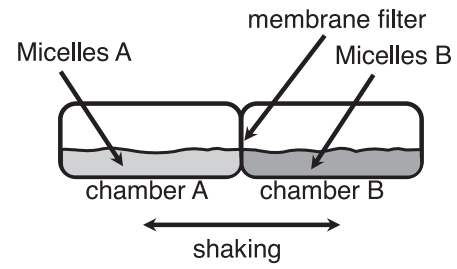

Fig. 2. Schematic view of the apparatus for measurement of the diffusion rate of micelles. Micelles A and $\mathrm{B}$ were placed into chamber A and chamber B, respectively. Chambers A and B were separated by a membrane filter and incubated at $37^{\circ} \mathrm{C}$ in a rocking water bath for 4 or $8 \mathrm{~h}$. See "Materials and Methods" for more information.

micelles was examined according to a method described previously $(8-10)$. A lipid emulsion containing $5 \mathrm{mM}$ MO, $10 \mathrm{~mm}$ oleic acid, $10 \mathrm{~mm} \mathrm{TO}, 5 \mathrm{~mm}$ PC, $10 \mathrm{~mm}$ cholesterol, and $6.0 \mathrm{~mm}$ sodium taurocholate, with or without various amounts of RMD or maltodextrin, was prepared in $150 \mathrm{~mm}$ sodium phosphate buffer ( $\mathrm{pH}$ 7.0) by sonication. After incubation at $37^{\circ} \mathrm{C}$ for $24 \mathrm{~h}$, an aliquot of the emulsion was transferred to a Beckman ultracentrifuge tube and subjected to ultracentrifugation at $100,000 \times g$ for $1 \mathrm{~h}$ to separate bile salt micelles at $37^{\circ} \mathrm{C}$.

Lipid analyses. Total lipids in emulsion and micelles were extracted in 20 volumes of chloroform/methanol $(2: 1, \mathrm{v} / \mathrm{v})$ by the method of Folch et al. (11). An aliquot of extracted lipids was transferred to a test tube containing pentadecanoic acid, (which was used as an internal standard) and subjected to analyses of total fatty acids. Another aliquot of the extracted lipids was subjected to fractionation into MO, oleic acid, TO, and PC by thin layer chromatography (Merck Co. Ltd., Japan) using diethyl ether/benzene/ethanol/acetic acid $(40: 50: 2: 0.2, \mathrm{v} / \mathrm{v} / \mathrm{v} / \mathrm{v})$ as a developing solvent. Each lipid fraction was scraped off and transferred to a test tube containing pentadecanoic acid as an internal standard. Total lipids from the emulsion and micelles and each lipid fraction scraped from the TLC plate were transmethylated with $\mathrm{H}_{2} \mathrm{SO}_{4}$ /ethanol $(2: 230$, v/v), and analyzed by gas chromatography (Omegawax 320, Sigma-Aldrich, Tokyo, Japan). The amount of micellar solubility was expressed as a percentage of the amounts of oleic acid in bile salt micelles and in each lipid fraction relative to those in the emulsion.

Quantification of sodium taurocholate in the emulsion and micelles. An aliquot of emulsion and micelles was used for sodium taurocholate analysis according to the method of Perwaiz et al. (12). Briefly, to the emulsion and micelles, 23-nor- $5 \beta$-cholanic acid was added as an internal standard; then, lipids were hydrolyzed and taurocholic acid was deconjugated in $1.25 \mathrm{M} \mathrm{NaOH}$ ( $3 \mathrm{~mL}$ ) at $120^{\circ} \mathrm{C}$ for $6 \mathrm{~h}$. After extraction of unsaponifiable matter and acidification of the remaining solution, released cholic acid was extracted. The bile acid was methylated with diazomethane and then trimethylsilylated. The derivative was subjected to gas chromatography analysis (SPB-1 column, Spelco). The amount of sodium taurocholate in micelles was expressed as a percentage of the amount of sodium taurocholate contained in micelles relative to those in emulsion.

Measurement of diffusion of bile salt micelles in vitro. Two kinds of bile salt micellar solutions were prepared according to the method of Ikeda et al. $(8,9)$. The first micellar solution contained $6.6 \mathrm{~mm}$ sodium taurocholate, $0.6 \mathrm{~mm} \mathrm{PC}$, and $132 \mathrm{~mm} \mathrm{NaCl}$ in $15 \mathrm{~mm}$ sodium phosphate buffer $\mathrm{pH} 7.4$ (Micelles A). The second micellar solution contained $6.6 \mathrm{~mm}$ sodium taurocholate, $0.6 \mathrm{~mm}$ PC, $1 \mathrm{~mm}$ oleic acid, $0.5 \mathrm{~mm} \mathrm{MO}$, and $100 \mu \mathrm{M}$ cholesterol (Micelles B) in $15 \mathrm{~mm}$ sodium phosphate buffer $\mathrm{pH}$ 7.4. In the control group, RMD or maltodextrin was not added and in the RMD or maltodextrin group, RMD or maltodextrin was added to both Micelles $\mathrm{A}$ and $\mathrm{B}$. These micellar solutions were prepared by sonication, flushed with argon gas, sealed, and kept at $37^{\circ} \mathrm{C}$ for $24 \mathrm{~h}$. A simple apparatus was designed to mimic the diffusion of bile salt micelles to the apical surface of 


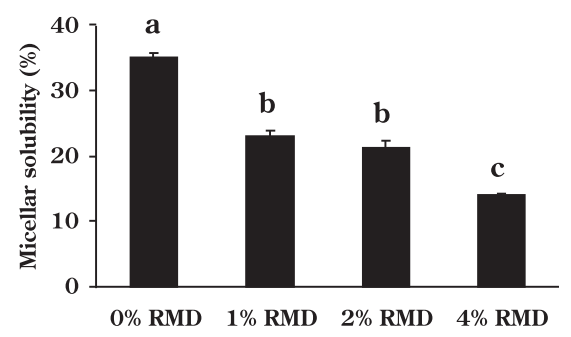

Fig. 3. Effects of resistant maltodextrin (RMD) on micellar solubility of total lipids, containing PC, MO, oleic acid, and TO, estimated as the total amount of oleic acid. PC, phosphatidylcholine; OA, oleic acid; MO, monooleoylglycerol; TO, trioleoylglycerol. Data are means \pm SE, $n=5$. Values with different letters are significantly different $(p<0.05)$.

intestinal epithelial cells in vitro (Fig. 2). This apparatus had two cylindrical glass chambers (chambers A and B, the volume was $15 \mathrm{~cm}^{3}$ each) separated by a $0.45-\mu \mathrm{m}$ membrane filter (HV, Millipore, Tokyo, Japan). The effective filter diameter was $15 \mathrm{~mm}$. Micelles A and B $(5 \mathrm{~mL}$ each) were transferred to chambers A and B, respectively. In the RMD or maltodextrin groups, RMD or maltodextrin was added to Micelles A and B. Then, the apparatus was longitudinally incubated for 4 and $8 \mathrm{~h}$ at $37^{\circ} \mathrm{C}$ (140 oscillations/min, shaking range $40 \mathrm{~mm}$ ). At the end of incubation, aliquots of Micelles A and B were subjected to analysis of lipids. Total lipids were extracted, and the concentrations of oleic acids and MO were measured as described above. If Micelles B in chamber B diffused to chamber A through the filter membrane, oleic acid and MO were expected to appear in chamber A. The diffusion rate (\%) was calculated as a percentage of the amount of oleic acid and MO appearing in chamber A at the end of incubation relative to that in chamber $\mathrm{B}$ before the incubation.

Measurement of uptake of micellar ${ }^{14} \mathrm{C}$-oleic acid by Caco-2 cells. Caco-2 cells (passage 40) were maintained on $10-\mathrm{cm}$ dishes at $37^{\circ} \mathrm{C}$ in an atmosphere containing $5 \% \mathrm{CO}_{2}$, in Dulbecco's Modified Eagle's Medium (DMEM, Sigma, Tokyo, Japan) supplemented with 10\% fetal bovine serum (FBS, Gibco, Invitrogen Co., Carlsbad, CA), $100 \mu \mathrm{g} / \mathrm{mL}$ streptomycin (Gibco), $100 \mathrm{IU} / \mathrm{mL}$ penicillin (Gibco), $2 \mathrm{~mm}$ L-glutamine (Gibco), $10 \mu \mathrm{g} / \mathrm{mL}$ insulin (Sigma), and 1\% non essential amino acids (MP Biochemicals Co., Santa Ana, CA). During semiconfluence, the cells were reseeded on a presoaked membrane filter coated with collagen (Transwell-COL, Corning Costar) and grown to full confluence, according to the method in our previous study (13). A bile salt micellar solution containing $0.5 \mathrm{mM} \mathrm{MO}, 1 \mathrm{~mm}$ oleic acid, $\left[1-{ }^{14} \mathrm{C}\right]$ oleic acid, $0.6 \mathrm{~mm}$ PC, $0.1 \mathrm{~mm}$ cholesterol, $6.6 \mathrm{~mm}$ sodium taurocholate, alone (control) or plus $40 \mathrm{mg} /$ mL RMD was prepared in DMEM by sonication. After filtration through a $0.22-\mu \mathrm{m}$ filter, $1.5 \mathrm{~mL}$ of the micellar solution supplemented with $10 \%$ FBS was added to the apical side of differentiated Caco- 2 cells and $2.5 \mathrm{~mL}$ DMEM was added to the basolateral side. The cells were incubated for 30 or $60 \mathrm{~min}$, respectively. At the end of

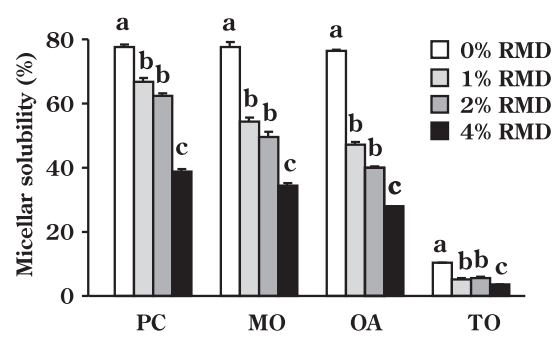

Fig. 4. Effects of resistant maltodextrin (RMD) on the solubility of individual lipid species in bile salt micelles. PC, phosphatidylcholine; OA, oleic acid; MO, monooleoylglycerol; TO, trioleoylglycerol. Data are means \pm SE, $n=5$. Values with different letters are significantly different $(p<0.05)$.

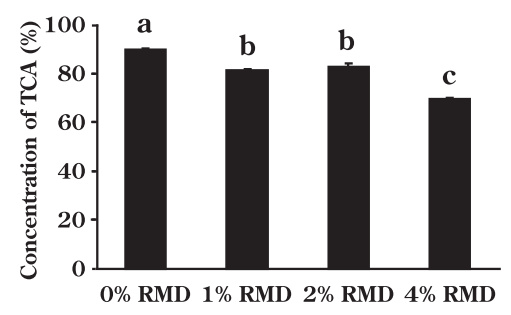

Fig. 5. Effects of resistant maltodextrin (RMD) on the concentration of taurocholic acid in bile salt micelles. TCA, taurocholic acid. Data are means \pm SE, $n=5$. Values with different letters are significantly different $(p<0.05)$.

the incubations, the solutions in the apical and basolateral compartments were collected and the cells were washed with PBS and then scraped off and lysed in tryp$\sin$ at $37^{\circ} \mathrm{C}$ for $10 \mathrm{~min}$ and then in a $0.1 \%$ Triton X-100 solution at $37^{\circ} \mathrm{C}$ for $30 \mathrm{~min}$. The radioactivity levels of the total lysates and in the solutions on both sides were measured with a liquid scintillation counter. The uptake rate (\%) was calculated as a percentage of the amount of the radioactivity incorporated into the cells at the end of the incubation relative to that in the bile salt micelles before the incubation.

Statistical analysis. Values are expressed as mean \pm SE. Data were tested i) by one-way analysis of variance (ANOVA) followed by the Tukey-Kramer test to identify differences among groups or ii) by Student's $t$-test within a pair of means. Differences were considered significant at $p<0.05$.

\section{RESULTS}

Effects of RMD and maltodextrin on micellar solubility of lipids in vitro

Figure 3 shows the effects of RMD on micellar solubility of total lipids including PC, MO, oleic acid, and TO estimated as the total amount of oleic acid. The total amount of oleic acid incorporated into the micellar solution was $35.0 \pm 0.7 \%$ of that in the emulsion with $0 \%$ RMD (Fig. 3). Percentages of oleic acid incorporated into the micelles dose-dependently decreased with the addi- 
tion of RMD. Almost the same tendency was observed for solubility of individual lipids, MO, oleic acid, TO, and PC, as shown in Fig. 4. The solubility of sodium taurocholate significantly and dose-dependently decreased with the addition of RMD, as shown in Fig. 5.

We also compared the effects of RMD with those of maltodextrin on the micellar solubility of lipids estimated as the amount of total oleic acid. Maltodextrin reduced micellar solubility of total lipids as in the case of RMD (Fig. 6).

Effects of RMD and maltodextrin on diffusion of bile salt micelles in vitro

The diffusion rates of oleic acid from Micelles B to Micelles A after 4 and $8 \mathrm{~h}$ of incubation in the RMDcontaining micelles were significantly lower than those in the control at both 4 and $8 \mathrm{~h}$ (Fig. 7A). The addition of maltodextrin had no significant effect on the diffusion

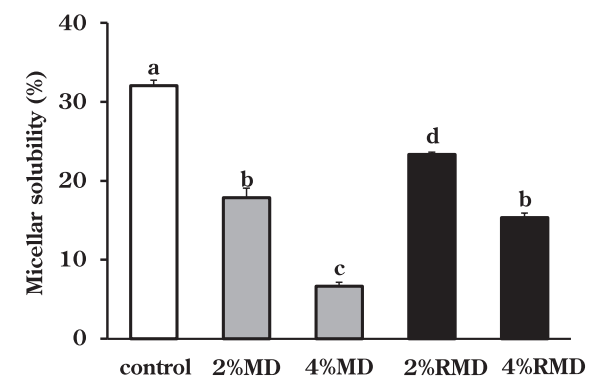

Fig. 6. Comparison of effects of resistant maltodextrin (RMD) and maltodextrin (MD) on micellar solubility of total lipids, containing PC, MO, oleic acid, and TO, estimated as the total amount of oleic acid. PC, phosphatidylcholine; OA, oleic acid; MO, monooleoylglycerol; TO, trioleoylglycerol. Data are means \pm SE, $n=5$. Values with different letters are significantly different $(p<0.05)$. rate of oleic acid after $4 \mathrm{~h}$ of incubation. However, the diffusion rate after $8 \mathrm{~h}$ of incubation was significantly lower in maltodextrin-containing micelles as compared with the control micelles (Fig. 7C). Similar results were also observed in the case of MO for both RMD (Fig. 7B) and maltodextrin (Fig. 7D).

Effects of RMD on uptake of radiolabeled oleic acid by Caco-2 cells

As shown in Fig. 8, the incorporation of $\left[1-{ }^{14} \mathrm{C}\right]$ oleic acid into Caco-2 cells was significantly smaller in the RMD-containing micelles than that in the control micelles after both 30- and 60-min incubation. Less than $0.65 \%$ of the radioactivity added as micellar solutions was secreted to the basolateral side, and no difference was observed between the control and RMD-containing micelles. It is known that Caco- 2 cells have an extremely limited ability to secrete chylomicron to the basolateral side (14).

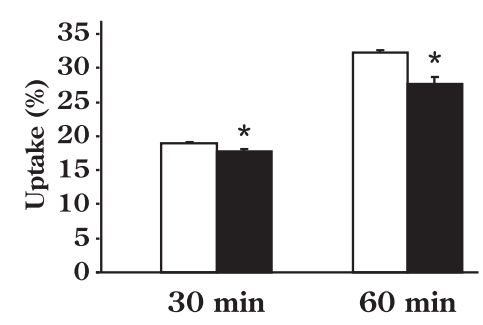

Fig. 8. Effects of resistant maltodextrin (RMD) on uptake of $\left[1-{ }^{14} \mathrm{C}\right]$ oleic acid in bile salt micelles by Caco- 2 cells. Bile salt micelles containing $\left[1-{ }^{14} \mathrm{C}\right]$ oleic acid were added to the apical side of Caco- 2 cells with (filled bars) or without (open bars) RMD $(40 \mathrm{mg} / \mathrm{mL})$. Data are means \pm SE of 5 measurements. ${ }^{*} p<0.05$ compared to the control group.

\section{A}

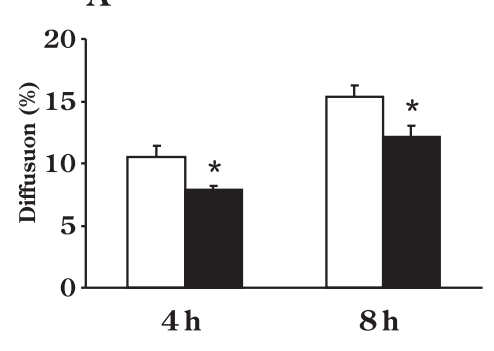

C

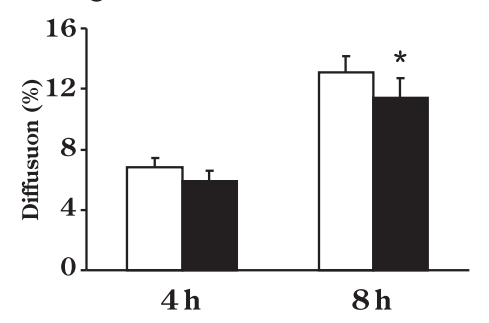

B

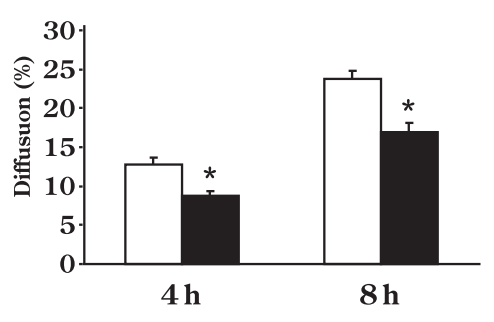

D

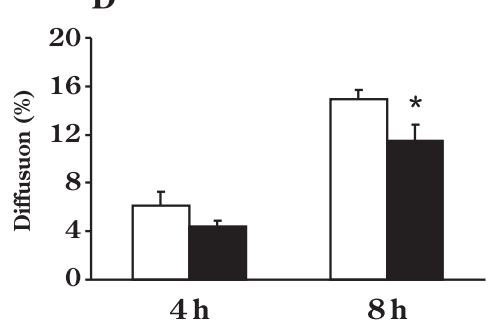

Fig. 7. Effects of resistant maltodextrin (RMD) and maltodextrin (MD) on diffusion of micelles from chamber A to B in vitro. A: Effects of RMD on the percentage of micelle diffusion, expressed as an estimate of oleic acid. B: Effects of RMD on the percentage of micelle diffusion, estimated as MO. C: Effects of MD on the percentage of micelle diffusion, estimated as oleic acid. D: Effects of MD on the percentage of micelle diffusion, estimated as MO. Open bars: RMD was not added (control group). Filled bars: RMD was added at $40 \mathrm{mg} / \mathrm{mL}$ (RMD group). MO, monooleoylglycerol. Data are means \pm SE of 6 measurements. ${ }^{*} p<0.05$ compared to the control group. 


\section{DISCUSSION}

Kishimoto et al. reported that dietary RMD suppresses postprandial hypertriacylglycerolemia in rats and humans $(5,6)$. Because RMD is indigestible and persists in the small intestine, there is high probability that RMD influences processes of digestion and absorption of dietary triacylglycerols in the small intestine. After ingestion of dietary triacylglycerols, they are digested by pancreatic lipase. Kishimoto et al. showed that RMD does not inhibit pancreatic lipase activity in vitro (6). Digestion products of dietary triacylglycerols, fatty acids, and MO are incorporated into bile salt micelles in the intestine. It is known that their solubilization and incorporation into bile salt micelles is essential for their effective absorption in the intestine (7). In the present study, to explore the mechanisms of action of RMD on the absorption of dietary triacylglycerols, an experiment was performed in vitro to determine the solubility of lipids in bile salt micelles. As a result, RMD significantly decreased the solubility of various lipid components in the bile salt micelles, and the effect was dose-dependent (Figs. 3 and 4). Because micellar solubilization of various lipids may be essential for their effective absorption, this can be one of the reasons why RMD suppresses intestinal absorption of triacylglycerols.

When the effect of RMD was compared with that of maltodextrin, both had a similar suppressive effect on micellar solubility of lipids in vitro (Fig. 6). It is known that RMD has three-dimensional tree structures (2), in which $1 \rightarrow 4,1 \rightarrow 6,1 \rightarrow 3$, and $1 \rightarrow 2$ glycosidic bonds are formed during its manufacture. On the other hand, in maltodextrin, the $1 \rightarrow 4$ glycosidic bond is predominant, and several percent of the $1 \rightarrow 6$ glycosidic bond is also present. Although three-dimensional structures are presumed to be different between RMD and maltodextrin, similar suppressive effects on micellar solubility of lipids were observed here. Our results suggest that the presence of the bulk of RMD and maltodextrin, rather than their three-dimensional structures, drives the suppressive effects on micellar solubility of lipids. We believe that RMD and maltodextrin trap various lipids inside their tree structures and impede their incorporation into bile salt micelles. In our opinion, maltodextrin cannot exert its suppressive effects on lipid absorption in vivo, because it can be quickly digested in the intestine. In contrast, RMD is resistant to digestion and therefore persists in the gut and maintains its advantage.

We also showed that taurocholate concentration in the bile salt micelles was lower with the addition of RMD (Fig. 5), although this decrease was weaker than that in lipid components. It is known that some types of watersoluble dietary fiber have bile acid-binding capacity (15, 16). However, it has never been reported that RMD can bind bile acids. Therefore, we believe that taurocholic acid is also trapped inside the tree structure of RMD molecules. The reduction in micellar solubility of taurocholic acid by RMD was weaker in comparison with lipid components. The results suggest that there are some differences in the interactions of RMD with lipid compo- nents and taurocholic acid, in addition to differences in trapping inside the tree structure of RMD.

It is known that during intestinal digestion of dietary fat, the products of digestion of triacylglycerols by pancreatic lipase are continuously incorporated into bile salt micelles, which then penetrate the unstirred water layer [an aqueous diffusion barrier covering intestinal cell surface (7)], and the micelles diffuse to the surface of intestinal epithelial cells for uptake. The present study showed that RMD and maltodextrin reduce the diffusion rate of bile salt micelles across the membrane filter (Fig. 7), suggesting that RMD and maltodextrin disturb diffusion of bile salt micelles from the intestinal lumen to the surface of intestinal epithelial cells through the unstirred water layer. We also showed that RMD significantly suppresses the uptake of micellar $\left[1-{ }^{14} \mathrm{C}\right]$ oleic acid into Caco-2 cells (Fig. 8), which were shown to closely mimic the kinetics of absorption in the human intestine (17). It is likely that the smaller incorporation of ${ }^{14} \mathrm{C}$-oleic acid into Caco- 2 cells was caused by RMD's interference with diffusion of bile salt micelles. We believe that the phenomenon in the intestinal lumen can cause delay to lipid absorption and therefore can be a reason for the suppressive effect of RMD on postprandial hypertriacylglycerolemia. Our results also suggest that both RMD and maltodextrin disturb the diffusion of bile salt micelles to the surface of intestinal epithelial cells. Again, because maltodextrin is quickly digested in the intestinal lumen, we believe that it cannot influence the diffusion of bile salt micelles in vivo.

It has been reported that guar gum and konjac mannan delay intestinal glucose absorption, probably by affecting its diffusion in the intestine due to their high viscosity $(18,19)$. Although there is no information on the effects of water-soluble dietary fiber on diffusion of bile salt micelles, its viscosity can be a major determinant of the diffusion rate of the micelles. In this study, we showed for the first time that RMD suppresses diffusion of bile salt micelles although the viscosity of RMD is far lower than those of these soluble dietary fibers. We also speculate that RMD covers the surface of bile salt micelles and suppresses their diffusion. However, because no evidence is available, further research is necessary in this respect.

The present study suggests that the observed suppression of postprandial hypertriacylglycerolemia by dietary RMD $(5,6)$ can be caused by delayed absorption of lipids via suppression of micellar solubility of lipids and delayed diffusion of bile salt micelles to the surface of intestinal epithelial cells.

\section{REFERENCES}

1) Borén J, Matikainen N, Adiels M, Taskinen M-R. 2014. Postprandial hypertriglyceridemia as a coronary risk factor. Clin Chim Acta 431: 131-142.

2) Ohkuma K, Matsuda I, Katta Y, Hanno Y. 1990. Pyrolysis of starch and its digestibility by enzymes - characterization of resistant maltodextrin. J Jpn Soc Starch Sci 37: 107-114 (in Japanese).

3) Wakabayashi S, Satouchi M, Nogami Y, Ohkuma K, Matsuoka A. 1991. Effect of resistant maltodextrin on 
cholesterol metabolism in rat. J Jpn Soc Nutr Food Sci 44: 471-478.

4) Nagata J, Saito M. 2006. Effects of simultaneous intakes of resistant maltodextrin and diacylglycerol on lipid profiles in rats fed cholesterol diets. Nutrition 22: 395-400.

5) Kishimoto Y, Oga H, Tagami H, Okuma K, Gordon DT. 2007. Suppressive effect of resistant maltodextrin on postprandial blood triacylglycerol elevation. Eur J Nutr 46: 133-138.

6) Kishimoto Y, Yoshikawa Y, Miyazoto S, Oga H, Yamada T, Tagami H, Hashizume C, Yamamoto K. 2009. Effect of resistant maltodextrin on digestion and absorption of lipids. J Health Sci 55: 834-844.

7) Nordskog BK, Phan CT, Nutting DF, Tso P. 2001. An examination of the factors affecting intestinal lymphatic transport of dietary lipids. Adv Drug Deliv Rev 50: 21-44.

8) Hamada T, Goto H, Yamahira T, Sugawara T, Imaizumi K, Ikeda I. 2006. Solubility in and affinity for the bile salt micelle of plant sterols are important determinants of their intestinal absorption in rats. Lipids 41: 551-556.

9) Ikeda I, Imasato Y, Sasaki E, Nakayama M, Nagao H, Takeo T, Yayabe F, Sugano M. 1992. Tea catechins decrease micellar solubility and intestinal absorption of cholesterol in rats. Biochim Biophys Acta 1127: 141-146.

10) Ikeda I, Tanaka K, Sugano M, Vahouny GV, Gallo LL. 1988. Inhibition of cholesterol absorption in rats by plant sterols. J Lipid Res 29: 1573-1582.

11) Folch J, Lees M, Sloane Stanley GH. 1957. A simple method for the isolation and purification of total lipids from animal tissues. J Biol Chem 226: 497-509.
12) Perwaiz S, Tuchweber B, Mignault D, Gilat T, Yousef IM. 2001. Determination of bile acids in biological fluids by liquid chromatography-electrospray tandem mass spectrometry. J Lipid Res 42: 114-119.

13) Hamada T, Ikeda I, Takashima K, Kobayashi M, Kodama Y, Inoue T, Matsuoka R, Imaizumi K. 2005. Hydrolysis of micellar phosphatidylcholine accelerates cholesterol absorption in rats and Caco-2 cells. Biosci Biotechnol Biochem 69: 1726-1732.

14) Levy E, Mehran M, Seidman E. 1995. Caco-2 cells as a model for intestinal lipoprotein synthesis and secretion. FASEB J 9: 626-635.

15) Vahouny GV, Tombes R, Cassidy M, Kritchevsky D, Gallo LL. 1981. Dietary fibers: V. binding of bile salts, phospholipids and cholesterol from mixed micelles by bile acid sequestrants and dietary fibers. Proc Soc Exp Biol Med 166: 1012-1018.

16) Ebihara K, Schneeman BO. 1989. Interaction of bile acids, phospholipids, cholesterol and triglyceride with dietary fibers in small intestine of rats. J Nutr 119: 1100-1106.

17) Tsuzuki W. 2007. Absorption properties of micellar lipid metabolites into Caco-2 cells. Lipid 42: 613-619.

18) Johnson IT, Gee JM. 1981. Effect of gel-forming gums on the intestinal unstirred layer and sugar transport in vitro. Gut 22: 398-403.

19) Ebihara K, Masuhara R, Kiriyama S. 1981. Major determinants of plasma glucose-flattening activity of a water-soluble dietary fiber: Effects of konjac mannan on gastric emptying and intraluminal glucose diffusion. Nutr Rep Int 23: 1145-1156. 